\title{
Exciton absorption in semiconductor quantum wells driven by a strong intersubband pump field
}

\author{
Ansheng $\mathrm{Liu}^{*}$ and C. Z. Ning \\ NASA Ames Research Center, M/S N229-1, Moffett Field, CA 94035 \\ ${ }^{*}$ Department of Electrical Engineering, Arizona State University, Tempe, AZ 85287
}

July 29,1999

\begin{abstract}
Optical interband excitonic absorption of semiconductor quantum wells (QW's) driven by a coherent pump field is investigated based on semiconductor Bloch equations. The pump field has a photon energy close to the intersubband spacing between the first two conduction subbands in the QW's. An external weak optical field probes the interband transition. The excitonic effects and pump-induced population redistribution within the conduction subbands in the QW system are included. When the density of the electron-hole pairs in the QW structure is low, the pump field induces an Autler-Townes splitting of the exciton absorption spectrum. The split size and the peak positions of the absorption doublet depend not only on the pump frequency and intensity but also on the carrier density. As the density of the electron-hole pairs is increased, the split contrast (the ratio between the maximum and minimum values) is decreased because the exciton effect is suppressed at higher densities due to the many-body screening.
\end{abstract}

OCIS numbers: $190.5970,190.4720,270.1670,270.4180$ 


\section{1. introduction}

In the past few years there has been a lot of interest in the optical interband and intersubband responses of semiconductor quantum wells (QW's) driven by a strong pump field. ${ }^{1-10}$ These investigations are partly motivated by the intensive work on electromagnetically induced transparency (EIT) ${ }^{11}$ and gain without inversion (GWI) ${ }^{12-15}$ in atomic systems. Compared to the atomic systems, the semiconductor nanostructures such as QW's have an advantage of feasible control of their electronic and optical properties by use of band-gap engineering. This unique feature makes these semiconductor quantum structures attractive for optoelectronic device applications. Since semiconductor QW's are different from atomic systems in many aspects, whether one can obtain similar phenomena in these quantum structures to those of the atomic systems still remains to be explored. Previously, it was shown that the optical interband absorption of a three-subband (two conduction subbands and one valence subband) QW structure can be significantly reduced by applying a strong pump field to couple the two empty conduction subbands. ${ }^{3,4}$ Such a reduction in the probe absorption is due to the coherent pump-probe nonlinear interaction in the QW system. Under the condition that there is a population inversion between the upper and lower conduction subbands, it was also predicted that GWI for the interband probe field is possible in the intersubband-pumped three-subband QW system. ${ }^{7}$ The appearance of the GWI is mainly due to the strong exciton effects in the QW structure. Although such a prediction seems to be attractive, for a three-subband QW structure the population inversion between the two lowest conduction subbands is experimentally difficult to achieve. Nevertheless, this result suggests that the carrier distribution in the conduction band influences significantly the interband probe spectrum because of the pump-probe coherent interaction in the $\mathrm{QW}$ system.

In this paper we employ the semiconductor Bloch equations approach to study the exciton absorption of semiconductor quantum wells pumped by a strong laser beam. The influence of the carrier density on the exciton absorption of QW structures in the presence 
of intersubband pumping is investigated. The electron-hole pairs in the undoped QW structure are generated solely by electrical current injection, since the probe field is assumed to be weak so that the probe beam does not excite an appreciable portion of carriers in the system. The carrier density is assumed to be smaller than the Mott density so that the exciton effect is sufficiently strong in our QW system. Taking into account the pumping effect, the electron distribution in the conduction band is determined self-consistently from a set of rate equations in which both intrasubband and intersubband relaxation processes are included. For a GaAs/AIGaAs QW structure, we perform a detailed numerical calculation of the exciton absorption spectrum by varying various parameters such as the pump frequency, the pump intensity, and the carrier density. We find that the exciton absorption spectrum strongly depends on these parameters. We also show that the many-body screening effect significantly suppresses the influence of the pump field on the interband absorption spectrum of the QW structure.

Our paper is organized as follows. In Sec. 2 we present our theory of the exciton absorption in a QW structure with an intersubband coupling field based on semiconductor Bloch equations. ${ }^{16,17}$ Taking into account the coherent pump-probe interaction, we calculate the optical polarization of the QW for the probe field from a set of coupled semiconductor equations. We then define the probe field susceptibility response tensor (hence the optical absorption coefficient) of the QW driven by a pump field. In Sec. 3 we present a numerical study of the exciton absorption spectrum of a GaAs/AlGaAs QW. The carrier density effect on the exciton absorption line of the QW for different pump frequency and intensity is then investigated. Finally we give a conclusion in Sec. 4.

\section{Theory}

We consider a symmetric semiconductor QW structure with two electron subbands (labelled by 2 and 3 ) within the conduction band and one heavy-hole subband (labelled by 1 ) in the valence band, as shown in Fig. 1. The two conduction subbands are resonantly coupled by a 
strong pump field $\left(\vec{E}_{p}\right)$ with an angular frequency $\omega_{p}$. A weak signal field $\left(\vec{E}_{\omega}\right)$ of frequency $\omega$ probes the interband excitonic transition between the hole subband and the lowest conduction subband (subband 2). For the symmetric QW structure considered in this paper, the interband transition between the hole subband and the second conduction subband (subband 3 ) is dipole forbidden if the band mixing effect is neglected. Since the photon energy of the pump field is much smaller than the interband transition energy, the pump field does not create carriers or electron-hole pairs. The total electron and hole densities are controlled by electrical current injection. To describe the probe response of the intersubband-driven $Q W$ system, we employ the semiconductor Bloch equations (SBE) formalism. ${ }^{16,17}$ The many-body effect in the optical response of the QW system is treated under the Hartree-Fock approximation. Thus, the influence of the carrier density on the exciton absorption spectrum in the presence of an intersubband pump field can be conveniently included. Such an effect was not analyzed in the previous work. ${ }^{7,9}$ Under the rotating-wave approximation (RWA) and in the steady state, the coupled semiconductor Bloch equations for the diagonal (population distribution) and off-diagonal (polarization) matrix elements are given by ${ }^{10,16,17}$

$$
\begin{gathered}
{\left[\hbar \omega-\tilde{E}_{21}\left(k_{\|}\right)+i \Gamma_{12}\right] \rho_{21}^{\omega}\left(k_{\|}\right)+\left[1-f_{2}\left(k_{\|}\right)-f_{1}\left(k_{\|}\right)\right] \sum_{\vec{k}_{\|}^{\prime}} V_{21}\left(\left|\vec{k}_{\|}-\vec{k}_{\|}^{\prime}\right|\right) \rho_{21}^{\omega}\left(k_{\|}^{\prime}\right)} \\
=-\vec{\mu}_{21}\left(k_{\|}\right) \cdot \vec{E}_{\omega}\left[1-f_{2}\left(k_{\|}\right)-f_{1}\left(k_{\|}\right)\right]-\vec{\mu}_{32}^{*}\left(k_{\|}\right) \cdot \vec{E}_{p}^{*} \rho_{31}^{\omega+\omega_{p}}\left(k_{\|}\right) \\
{\left[\hbar\left(\omega+\omega_{p}\right)-\tilde{E}_{31}\left(k_{\|}\right)+i \Gamma_{13}\right] \rho_{31}^{\omega+\omega_{p}}\left(k_{\|}\right)+\left[1-f_{3}\left(k_{\|}\right)-f_{1}\left(k_{\|}\right)\right] \sum_{\vec{k}_{\|}^{\prime}} V_{31}\left(\left|\vec{k}_{\|}-\vec{k}_{\|}^{\prime}\right|\right) \rho_{31}^{\omega+\omega_{p}}\left(k_{\|}^{\prime}\right)} \\
=-\vec{\mu}_{32}\left(k_{\|}\right) \cdot \vec{E}_{p} \rho_{21}^{\omega}\left(k_{\|}\right)+\vec{\mu}_{21}\left(k_{\|}\right) \cdot \vec{E}_{\omega} \rho_{32}^{\omega_{p}}\left(k_{\|}\right) \\
{\left[\hbar \omega_{p}-\tilde{E}_{32}\left(k_{\|}\right)+i \Gamma_{23}\right] \rho_{32}^{\omega_{p}}\left(k_{\|}\right)-\left[f_{2}\left(k_{\|}\right)-f_{3}\left(k_{\|}\right)\right] \sum_{\vec{k}_{\|}^{\prime}} V_{32}\left(\left|\vec{k}_{\|}-\vec{k}_{\|}^{\prime}\right|\right) \rho_{32}^{\omega_{p}}\left(k_{\|}^{\prime}\right)} \\
=-\vec{\mu}_{32}\left(k_{\|}\right) \cdot \vec{E}_{p}\left[f_{2}\left(k_{\|}\right)-f_{3}\left(k_{\|}\right)\right] \\
-\frac{2}{\hbar} \operatorname{Im}\left[\vec{\mu}_{32}^{*}\left(k_{\|}\right) \cdot \vec{E}_{p}^{*} \rho_{32}^{\omega_{p}}\left(k_{\|}\right)\right]-\frac{f_{2}\left(k_{\|}\right)-f_{2}^{\mathrm{F}}\left(k_{\|}\right)}{T_{c c}}+\frac{f_{3}\left(k_{\|}\right)}{\tau_{32}}=0
\end{gathered}
$$




$$
\frac{2}{\hbar} \operatorname{Im}\left[\vec{\mu}_{32}^{*}\left(k_{\|}\right) \cdot \vec{E}_{p}^{*} \rho_{32}^{\omega_{p}}\left(k_{\|}\right)\right]-\frac{f_{3}\left(k_{\|}\right)-f_{3}^{F}\left(k_{\|}\right)}{T_{c c}}-\frac{f_{3}\left(k_{\|}\right)}{\tau_{32}}=0
$$

In the above equations,

$$
V_{i j}(q)=\frac{e^{2}}{2 \epsilon_{0} \epsilon_{B} \epsilon(q) q S} \iint \psi_{i}^{2}(z) e^{-q\left|z-z^{\prime}\right|} \psi_{j}^{2}\left(z^{\prime}\right) d z^{\prime} d z
$$

is the screened Coulomb matrix element that describes the carrier-carrier Coulomb interactions, $\psi_{i}(z)$ and $\psi_{j}(z)$ being the envelope wave functions for subband $i$ and subband $j$, respectively (the QW growth direction is assumed to be along the $z$ axis). In Eq. (6) $\epsilon_{B}$ is the background dielectric constant of the QW structure and $S$ is the (normalization) crosssection area of the QW structure. The many-body screening effect is taken into account by introducing a screening factor $\epsilon(q)$ in Eq. (6) under the plasmon-pole approximation. ${ }^{18} \Gamma_{i j}$ in Eqs. (1)-(3) is the line broadening factor for both intersubband and interband/exciton transitions. The function $\bar{E}_{i j}\left(k_{\|}\right)$is the renormalized single-particle energy separation between subbands $i$ and $j$. For interband transitions,

$\tilde{E}_{i j}\left(k_{\|}\right)=E_{i}\left(k_{\|}\right)+E_{j}\left(k_{\|}\right)-\sum_{\vec{k}_{\|}^{\prime}} V_{i j}\left(\left|\vec{k}-\vec{k}^{\prime}\right|\right)\left[f_{i}\left(k_{\|}^{\prime}\right)+f_{j}\left(k_{\|}^{\prime}\right)\right]-\Delta E_{g}^{\mathrm{CH}}$,

where $\Delta E_{g}^{\mathrm{CH}}$ is the Coulomb hole contribution to the band gap renormalization. ${ }^{18}$ For the intersubband transition energy,

$\tilde{E}_{i j}\left(k_{\|}\right)=E_{i}\left(k_{\|}\right)-E_{j}\left(k_{\|}\right)-\sum_{\vec{k}_{\|}^{\prime}} V_{i j}\left(\left|\vec{k}-\vec{k}^{\prime}\right|\right)\left[f_{i}\left(k_{\|}^{\prime}\right)-f_{j}\left(k_{\|}^{\prime}\right)\right]$

Note that in writing the above semiconductor Bloch equations we have adopted the electric dipole approximation to account for the light-QW interaction, and $\vec{\mu}_{i j}\left(k_{\|}\right)$denotes the dipole matrix element. For the interband transition, the dipole moment is given by $\vec{\mu}_{i j}\left(k_{\|}\right)=\frac{e \hbar \mathcal{O}_{i j} \vec{P}_{c v}}{i m_{0} E_{i j}\left(k_{\|}\right)}$ where $\mathcal{O}_{i j}=\int \psi_{i}^{*}(z) \psi_{j}(z) d z$ is the overlap integral between the conduction and valence envelope wave functions and $\vec{P}_{c v}$ is the Kane's interband momentum matrix element. In Eqs. (4) and (5), $T_{c c}$ and $\tau_{32}$ are the intrasubband carrier-carrier scattering time and intersubband 
relaxation time, respectively. Neglecting the band mixing effect, the intersubband dipole moment $\vec{\mu}_{32}\left(k_{\|}\right)=\vec{\mu}_{32}$ is $k_{\|}$independent and along the QW growth direction ( $z$ axis), and the magnitude is $\mu_{32}=e \int \psi_{3}^{*}(z) z \psi_{2}(z) d z$. The quantities $f_{i}^{\mathrm{F}}\left(k_{\|}\right)(i=2,3)$ in Eqs. (4) and (5) are considered to be the quasi-equilibrium distribution functions, which are related to the Fermi-Dirac functions $f_{i}^{(0)}\left(k_{\|}\right)(i=2,3)$ in the absence of pump field via $f_{2}^{F}\left(k_{\|}\right)=f_{2}^{(0)}\left(k_{\|}\right)-\frac{T_{c c}}{\tau_{32}} f_{3}^{(0)}\left(k_{\|}\right)$,

and

$f_{3}^{\mathrm{F}}\left(k_{\|}\right)=\left(1+\frac{T_{c c}}{\tau_{32}}\right) f_{3}^{(0)}\left(k_{\|}\right)$.

Because the signal field is weak and the pump field does not resonanly couple the hole subbands, the hole distribution function $f_{1}\left(k_{\|}\right)$is simply the thermal equilibrium FermiDirac function in the absence of the pump beam.

For a given pump intensity and carrier density in the $\mathrm{QW}$ structure, we determine first the electron distribution function for each conduction subband from Eqs. (3)-(5) using Eqs. (10) and (11). To simplify the numerical calculation, we neglect the many-body effect on the pump-induced change in the subband population distribution as in the previous work. ${ }^{10}$ Including such an effect does not modify our results qualitatively. Then we solve $\rho_{21}^{\omega}\left(k_{\|}\right)$ from Eqs. (1) and (2). In our calculations, 201 equal-distant $k_{\|}$values are used. We then calculate the probe-induced polarization $\vec{P}(\omega)$ and define a susceptibility response tensor, $\overleftrightarrow{\chi}(\omega)$, for the signal field through

$\vec{P}(\omega)=\frac{2}{L_{\mathrm{w}}} \iint \frac{d^{2} k_{\|}}{(2 \pi)^{2}} \vec{\mu}_{21}^{*} \rho_{21}^{\omega}\left(k_{\|}\right)=\epsilon_{0} \overleftrightarrow{\chi}(\omega) \cdot \vec{E}_{\omega}$,

where $L_{\mathrm{w}}$ denotes the quantum well width and the factor of 2 accounts for the spin degeneracy. Note from Eqs. (1) and (2) that $\rho_{21}^{\omega}\left(k_{\|}\right)$is linearly proportional to the amplitude of the probe field. Therefore, the susceptibility tensor defined in Eq. (12) is independent of the intensity of the signal field, as it should be. However, it depends on the pump intensity through the pump induced change in the electron distribution functions within the conduction band as well as the pump-probe coherent interaction. The pump-probe electromagnetic 
coupling is manifested in the last term on the right-hand side of Eq. (1) as well as in the two terms on the right-hand side of Eq. (2). The pump field is assumed to be polarized along the quantum well growth direction in order to induce effectively the intersubband transition and the probe field is polarized parallel to the QW interfaces because the dipole moment of heavy hole to electron subband transition is dominant in such a polarization. The absorption coefficient is proportional to the imaginary part of the susceptibility.

\section{3. numerical results and discussions}

In this section we present a detailed numerical study on the signal-field absorption spectrum of a symmetric $\mathrm{GaAs} / \mathrm{Al}_{0.3} \mathrm{Ga}_{0.7} \mathrm{As} \mathrm{QW}$. Our attention is especially focused on the influence of the electron-hole density on the absorption spectrum of the QW structure in the presence of a strong pump field. For our numerical calculations, we choose a GaAs well width of 100 $\AA$. The band offset is assumed to follow the $60 \%-40 \%$ rule. $\Gamma_{12}=\Gamma_{23}=3.0 \mathrm{meV}, \Gamma_{13}=1.0$ $\mathrm{meV}, T_{c c}=0.5 \mathrm{ps}$, and $\tau_{32}=1.0 \mathrm{ps}$. Note that we use a smaller line broadening of $1.0 \mathrm{meV}$ for the interband transition between the valence suband and the second conduction subband because this transition is electric-dipole forbidden. The electron and heavy hole effective masses are $m_{e}=0.067 m_{0}$ and $m_{h}=0.47 m_{0}$, respectively. The subband eigenenergies and the corresponding wave functions for our QW structure are calculated from the effectivemass Schrödinger equation. For simplicity we neglect the band nonparabolicity effect for both the valence and conduction subbands and assume an equal effective mass for the two conduction subbands. Therefore, the intersubband separation $\left(E_{32}\right)$ between subband 3 and subband 2 is independent of the in-plane wave vector $\vec{k}_{\|}$. For the TE polarization of the probe field, the interband momentum matrix element is given by $P_{c v}=p_{0} / \sqrt{2}$ where $2 p_{0}^{2} / m_{0}=25 \mathrm{eV}$ is used. In all calculations we use a temperture of $77 \mathrm{~K}$.

To understand how a strong intersubband pump field influences the exciton absorption spectrum of our QW system and how the exciton absorption spectrum depends on the parameters such as the pump frequency, the pump intensity, and the carrier density, it 
is instructive to understand the results in the absence of the pump field. In Fig. 2 we show the probe absorption spectra of our QW structure for different electron-hole densities, i.e. $1 \times 10^{10}, 5 \times 10^{10}, 1 \times 10^{11}$, and $2 \times 10^{11} \mathrm{~cm}^{-2}$ without the pumping field. Fig. 2 shows that in the low carrier density case there is a sharp exciton absorption peak below the $\mathrm{QW}$ interband edge, as expected. The broad stair-like absorption spectrum in the high-frequency side of the exciton peak is due to continuum interband transitions. As the electron-hole density is increased, the exciton absorption line is slightly blueshifted although the band gap renormalization leads to a redshift and its peak value is decreased. This is because the screening length of the QW structure is decreased with an increase in the carrier density. Therefore the electron-hole Coulomb interaction becomes weaker and the exciton binding energy becomes smaller as well. The decrease in the exciton binding energy slightly exceeds the band gap shrinkage at the carrier densities used in Fig. 2 so that there is a net blueshift of the exciton absorption line. It is also interesting to note from Fig. 2 that the exciton line width is obviously broadened with increasing carrier density although a fixed value (3 $\mathrm{meV}$ ) for the line broadening is used. The reason is that the exciton effects become less important and the continuum interband transitions begin to dominate the contributions to the absorption spectrum when the exciton density is increased.

Now let us study the effect of the pump field on the exciton absorption line of the QW structure for different pump frequencies and intensities. In Fig. 3 we show the probe absorption spectra of the QW structure at the pump photon energy of $\hbar \omega_{p}=E_{32}$ for different pump intensities, namely $0,0.5,1.0,1.5$ and $2.0 \mathrm{MW} / \mathrm{cm}^{2}$. The carrier density used in the calculation is $5 \times 10^{10} \mathrm{~cm}^{-2}$. Note that, for clarity, we shift the absorption spectra vertically for nonzero pump intensities. We can see from Fig. 3 that applying a pump field to the QW structure leads to a splitting of the exciton absorption line. Such a split is analogous to the well-known Autler-Townes splitting of the atomic absorption line. Depending upon the probe frequency, the absorption coefficient is either decreased or increased with an increase in the pump intensity. In Fig. 4 we display the absorption coefficient as a function of the pump intensity at the probe photon energy equal to the exciton peak energy without the pump 
field. We see from Fig. 4 that, although the pump field reduces significantly the absorption coefficient, due to the continuum interband contribution to the optical absorption, the EIT in the QW structure is hardly achieved. Fig. 3 also shows that the splitting is increased with an increase in the pump intensity. The pump intensity dependence of the split energy is displayed in Fig. 5. We compared results in Fig. 5 with those obtained for atomic systems and found the split energy dependence on the pump intensity is quite similar for the two different systems. We also note from Fig. 3 that the heights of the two absorption peaks are dependent on the pump intensity. These dependencies of the peak heights on the pump intensity are shown in Fig. 6. As the pump intensity is increased, the lower energy peak decreases while the higher energy peak increases in magnitude. This result is different from what one would expect from atomic systems. In the atomic system, the heights of the two peaks decrease with an increase in the pump intensity when the pump photon energy is exactly equal to the level spacing between the two upper states. Also, the absorption line is symmetrically splitted with respect to the peak in the absence of the pump field in this case. These differences (cf. Figs. 3 and 6) are mainly due to the fact that the pump field excites the exciton-exciton transition instead of the bare intersubband transition in the QW system due to the electron-hole correlation. Because the binding energy of the valence to first conduction subband exciton is somewhat larger than that of the valence to second conduction subband exciton, the exciton-exciton transition energy is a little bit larger than the intersubband separation without the exciton effects. Therefore, the pump photon energy $\hbar \omega_{p}=E_{32}$ is indeed less than the exciton-resonant intersubband transition energy. In this case, we do not expect a symmetric splitting of the line splitting as for the atomic systems. Here we would like to stress that the band gap renormalizations for the two conduction subbands are slightly different. This results in a small increase in the intersubband transition energy, which also contributes to the asymmetrical split of the absorption spectra in Figs. 3 and 6.

To see more clearly the influence of the pump frequency on the exciton absorption spectrum, we show in Fig. 7 the exciton absorption coefficient as a function of the probe detuning at a pump intensity of $1 \mathrm{MW} / \mathrm{cm}^{2}$ for different values of the pump frequency detuning 
$\Delta=\hbar \omega_{p}-E_{32}$, namely $\Delta=-9,-6,-3,0,3,6$, and $9 \mathrm{meV}$. In Fig. 7 the electron-hole density is still chosen to be $5 \times 10^{10} \mathrm{~cm}^{-2}$. As the pump detuning is increased from zero to positive values, the higher-energy peak moves downwards and its peak height is gradually increased. However, the lower-energy peak is shifted towards the lower probe photon energy and its peak absorption coefficient is decreased. In particular, we note an exchange in the shape of the absorption doublet between the spectrum at $\Delta=0$ and the spectrum at $\Delta=3$ $\mathrm{meV}$. We therefore expect that the Autler-Townes splitting is symmetric when the pump detuning is bewteen 0 and $3 \mathrm{meV}$. This result confirms our explanation that the exciton effect slightly increases the intersubband energy separation between the first and second conduction subbands. When the pump detuning is further increased, the lower-energy peak becomes smaller and finally disappears at the lower probe photon energy side. This should be expected because when the detuning tends to infinity, the absorption spectrum should return to the result in the absence of the pump field, namely there is only one exciton peak in the absorption spectrum. On the other hand, as the pump frequency is decreased from $\Delta=0$, the lower-energy peak is shifted upwards and its peak height is increased. But the higher-energy peak is moved to the higher photon energy and its peak value is decreased. These results suggest that varying the intersubband pump frequency leads to not only the shift of the Autler-Townes doublet but also the absorption line shape.

Finally we study the influence of the carrier density on the pump-induced splitting of the exciton absorption line of the QW structure. In Fig. 8 we display the probe spectra at a pump intensity of $1 \mathrm{MW} / \mathrm{cm}^{2}$ for different carrier densities, i.e. $1 \times 10^{10}, 5 \times 10^{10}, 1 \times 10^{11}$, and $2 \times 10^{11} \mathrm{~cm}^{-2}$. In calculating Fig. 8 the pump frequency of $\hbar \omega_{p}=E_{32}$ is used. From Fig. 8 we note that, the pump field has a much stronger effect on the exciton absorption spectrum in the low carrier density case compared to the higher density case. As the exciton density is increased, both the size and contrast of the Autler-Townes splitting become smaller because the exciton effect becomes weaker. We have checked that, when the carrier density is so large that the exciton absorption peak almost disappears, the pump field has an even smaller effect on the absorption spectrum of the QW structure. These results suggest that the lower 
carrier density is favorable for observing the Autler-Townes split of the interband absorption line in the QW structure.

\section{4. conclusion}

In this paper, we have used the semiconductor Bloch equations to calculate optical interband absorption of semiconductor quantum wells driven by a coherent intersubband pump field. Both excitonic bound and continuum states contribute to the pump-probe spectrum of the QW system and are treated on an equal footing. The pump-induced population redistribution within conduction subbands in the QW system are included. When the electron-hole density is low, the pump field induces an Autler-Townes splitting of the exciton absorption spectrum. The split size and the peak positions of the absorption doublet depend not only on the pump frequency and intensity but also on the exciton density. As the carrier density is increased, we find that both the magnitude and contrast of the Autler-Townes splitting energy become smaller because of the carrier screening effect.

\section{ACKNOWLEDGMENTS}

The authors are indebted to professor S.-L. Chuang for his valuable comments on the manuscript. This work is partly supported by NASA Ames Research Center Director's Discretionary Fund. 


\section{REFERENCES}

1. Y. Zhao, D. Huang, and C. Wu, Opt. Lett. 19, 816 (1994).

2. A. Imamoglu and R. J. Ram, Opt. Lett. 19, 1744 (1994).

3. D. Huang, C. Wu, and Y. Zhao, J. Opt. Soc. Am. B 11, 2258 (1994).

4. D. S. Lee and K. J. Malloy, IEEE J. Quantum Electron. QE-30, 85 (1994).

5. Y. Zhao, D. Huang, and C. Wu, J. Nonlin. Opt. Phys. Mater. 4, 261 (1995).

6. J. B. Khurgin and E. Rosencher, IEEE J. Quantum Electron. QE-32, 1882 (1996).

7. D. S. Lee and K. J. Malloy, Phys. Rev. B 53, 15749 (1996).

8. A. Liu, Phys. Rev. A 56, 3206 (1997).

9. A. Neogi, Y. Takahashi, and H. Kawaguchi, IEEE J. Quantum Electron. QE-33, 2060 (1997).

10. A. Liu, J. Opt. Soc. Am. B 15, 1741 (1998).

11. See, for example, K. J. Boller, A. Imamoglu, and S. E. Harris, Phys. Rev. Lett. 66, 2593 (1991).

12. A. Nottelmann, C. Peters, and W. Lange, Phys. Rev. Lett. 70, 1783 (1993).

13. E. S. Fry, X. Li, D. E. Nikonov, G. G. Padmabandu, M. O. Scully, A. V. Smith, F. K. Tittel, C. Wang, S. R. Wilkinson, and S.-Y. Zhu, Phys. Rev. Lett. 70, 3235 (1993).

14. W. E. van der Veer, R. J. van Diest, A. Donszelmann, and H. B. van Linden van den Heuvell, Phys. Rev. Lett. 70, 3243 (1993).

15. A. S. Zibrov, M. D. Lukin, D. E. Nikonov, L. Hollberg, M. O. Scully, and V. L. Velichansky, Phys. Rev. Lett. 75, 1499 (1995).

16. S. Schmitt-Rink and D. S. Chemla, Phys. Rev. Lett. 57, 2752 (1986); S. Schmitt-Rink, 

D. S. Chemla, and H. Haug, Phys. Rev. B 37, 941 (1988).

17. H. Haug and S. W. Koch, Quantum Theory of the Optical and Electronic Properties of Semiconductors, 2nd ed. (World Scientific, Singapore, 1993).

18. W. W. Chow, S. W. Koch, and M. Sargent III, Semiconductor-Laser Physics (World Scientific, Singapore, 1995). 


\section{FIGURES}

Fig. 1. Pump-probe scheme in a three-subband $(|1, k\rangle,|2, k\rangle$, and $|3, k\rangle) \mathrm{QW}$ structure. The two conduction subbands are coupled by a coherent pump field of frequency $\omega_{p}$, and the interband transition is probed by a weak signal field with an angular frequency $\omega$.

Fig. 2. Probe absorption spectra of a GaAs/AlGaAs QW in the absence of the pump field for different exciton densities, i.e. $1 \times 10^{10}, 5 \times 10^{10}, 1 \times 10^{11}$, and $2 \times 10^{11} \mathrm{~cm}^{-2}$.

Fig. 3. Probe absorption spectra of a GaAs/AlGaAs QW at a pump photon energy of $\hbar \omega_{p}=E_{32}$ for different pump intensities, i.e. $0,0.5,1.0,1.5$, and $2 \mathrm{MW} / \mathrm{cm}^{2}$. The electron-hole density is $5 \times 10^{10} \mathrm{~cm}^{-2}$.

Fig. 4. Probe absorption coefficient as a function of the pump intensity at the probe photon energy equal to the exciton peak energy in the absence of the pump field. The pump photon energy is $\hbar \omega_{p}=E_{32}$, and the electron-hole density is $5 \times 10^{10} \mathrm{~cm}^{-2}$.

Fig. 5. Split energy as a function of the pump intensity. The pump photon energy is $\hbar \omega_{p}=E_{32}$. The electron-hole density is $5 \times 10^{10} \mathrm{~cm}^{-2}$.

Fig. 6. Heights of the Autler-Townes splitted exciton absorption peaks as a function of the pump intensity. The pump photon energy is $\hbar \omega_{p}=E_{32}$. The electron-hole density is $5 \times 10^{10} \mathrm{~cm}^{-2}$.

Fig. 7. Probe absorption spectra of a GaAs/AlGaAs QW at a pump intensity of $1 \mathrm{MW} / \mathrm{cm}^{2}$ for different pump frequency detuning $\Delta=\hbar \omega_{p}-E_{32}$, i.e. $\Delta=-9,-6,-3,0,3,6$, and $9 \mathrm{meV}$. The electron-hole density is $5 \times 10^{10} \mathrm{~cm}^{-2}$.

Fig. 8. Probe absorption spectra of a GaAs/AlGaAs QW at a pump intensity of $1 \mathrm{MW} / \mathrm{cm}^{2}$ for different exciton densities, namely $1 \times 10^{10}, 5 \times 10^{10}, 1 \times 10^{11}$, and $2 \times 10^{11} \mathrm{~cm}^{-2}$. The pump frequency is $\hbar \omega_{p}=E_{32}$. 


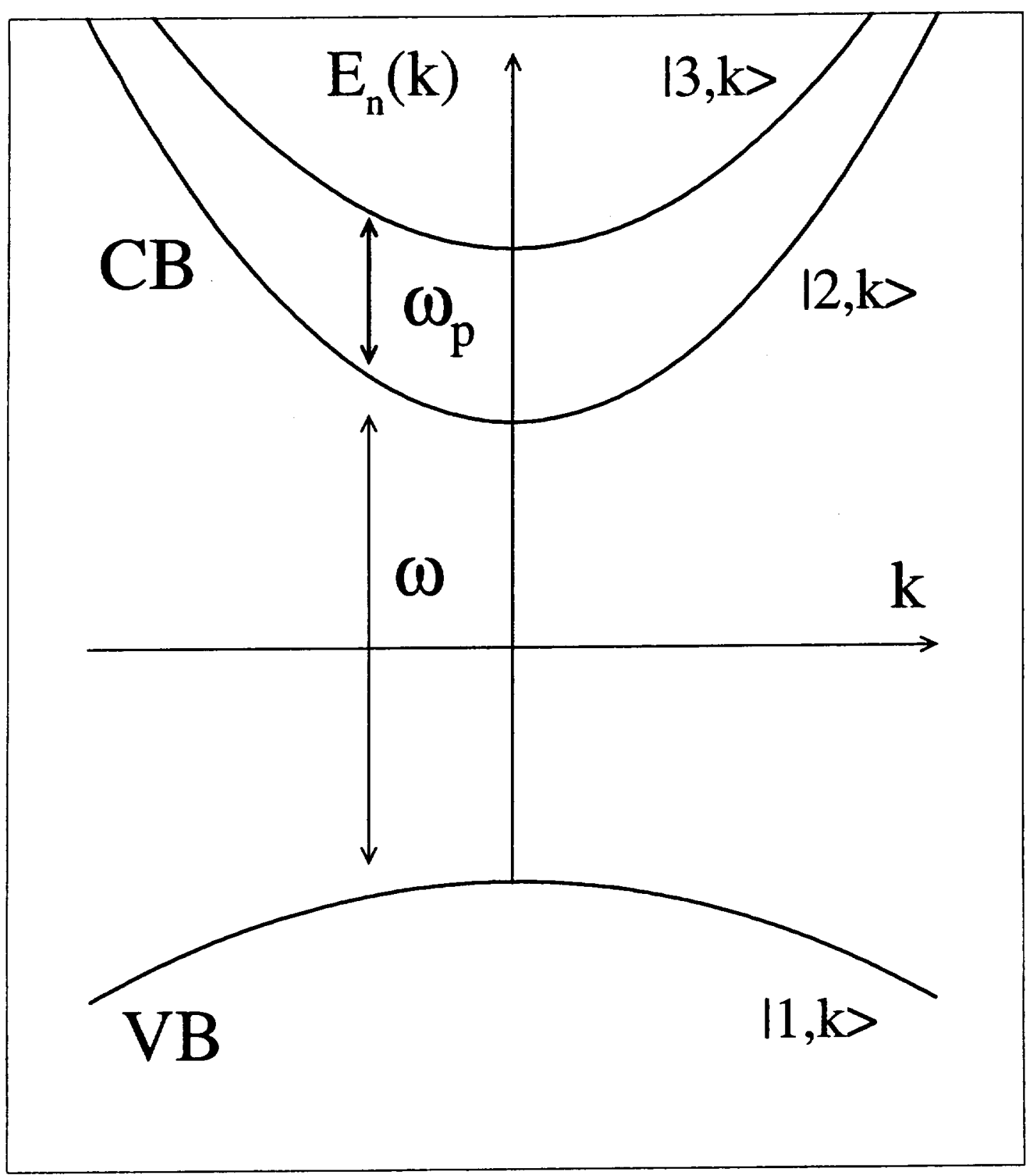

FIG. 1 


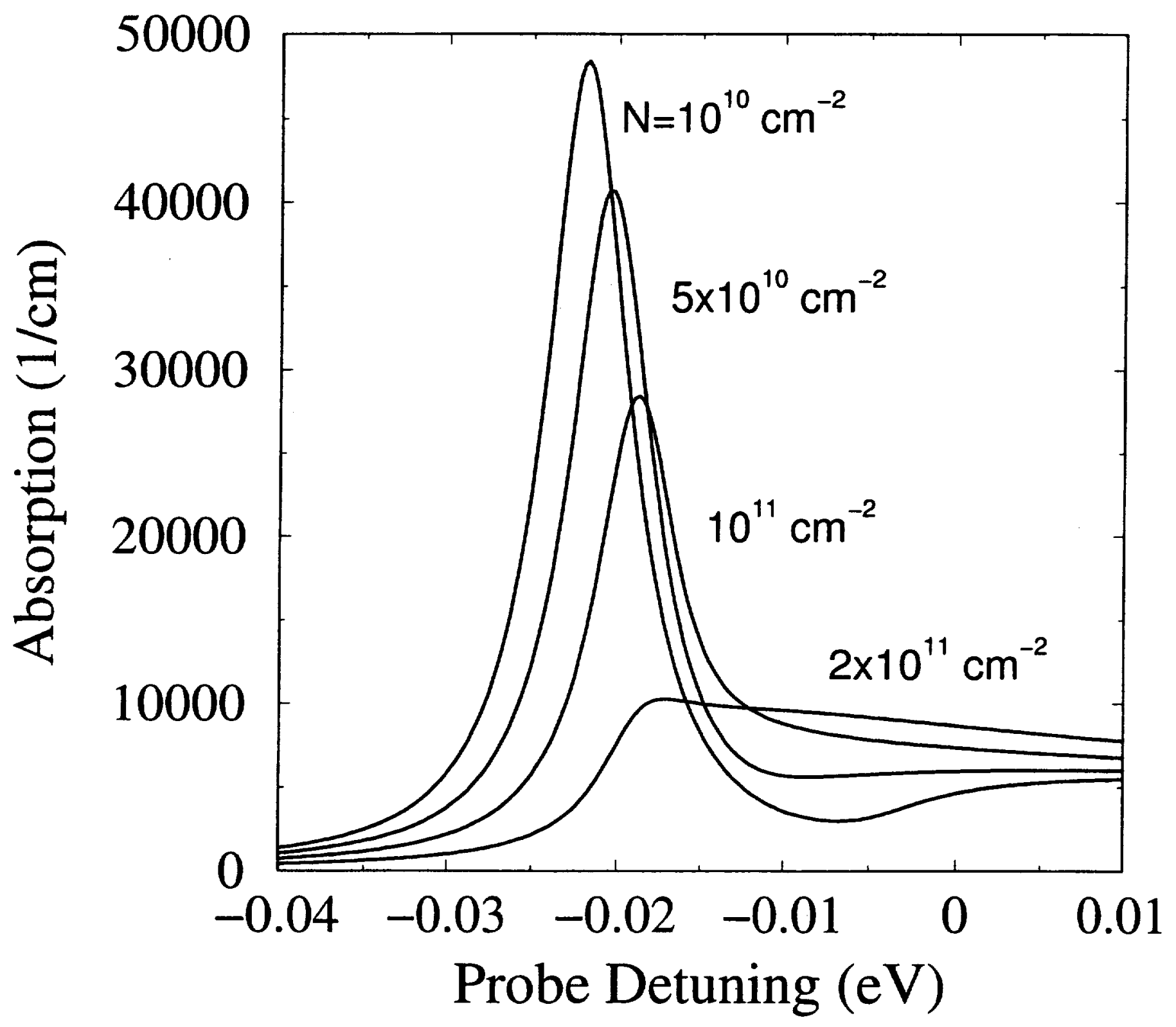

FIG. 2 


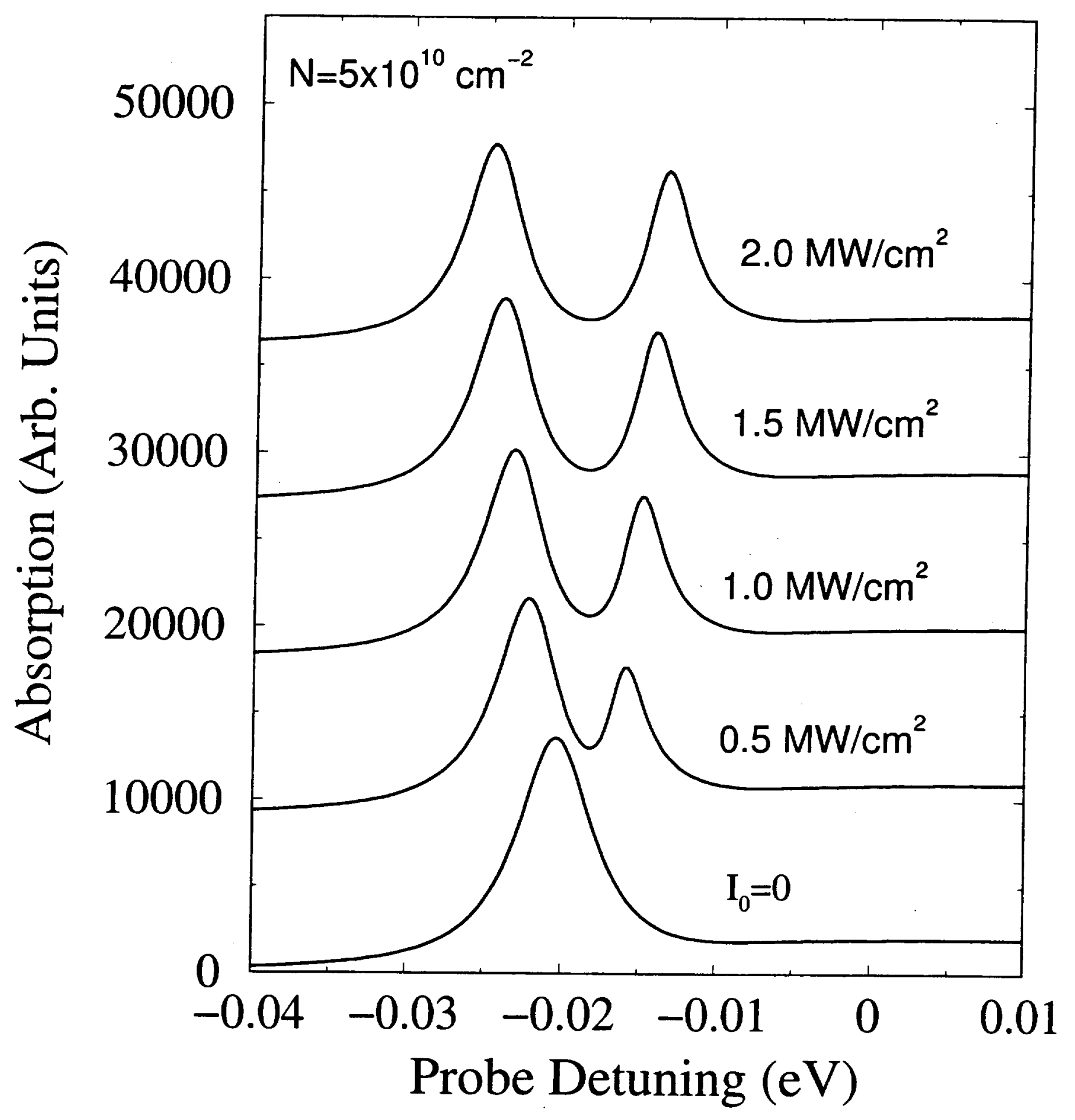

FIG. 3 


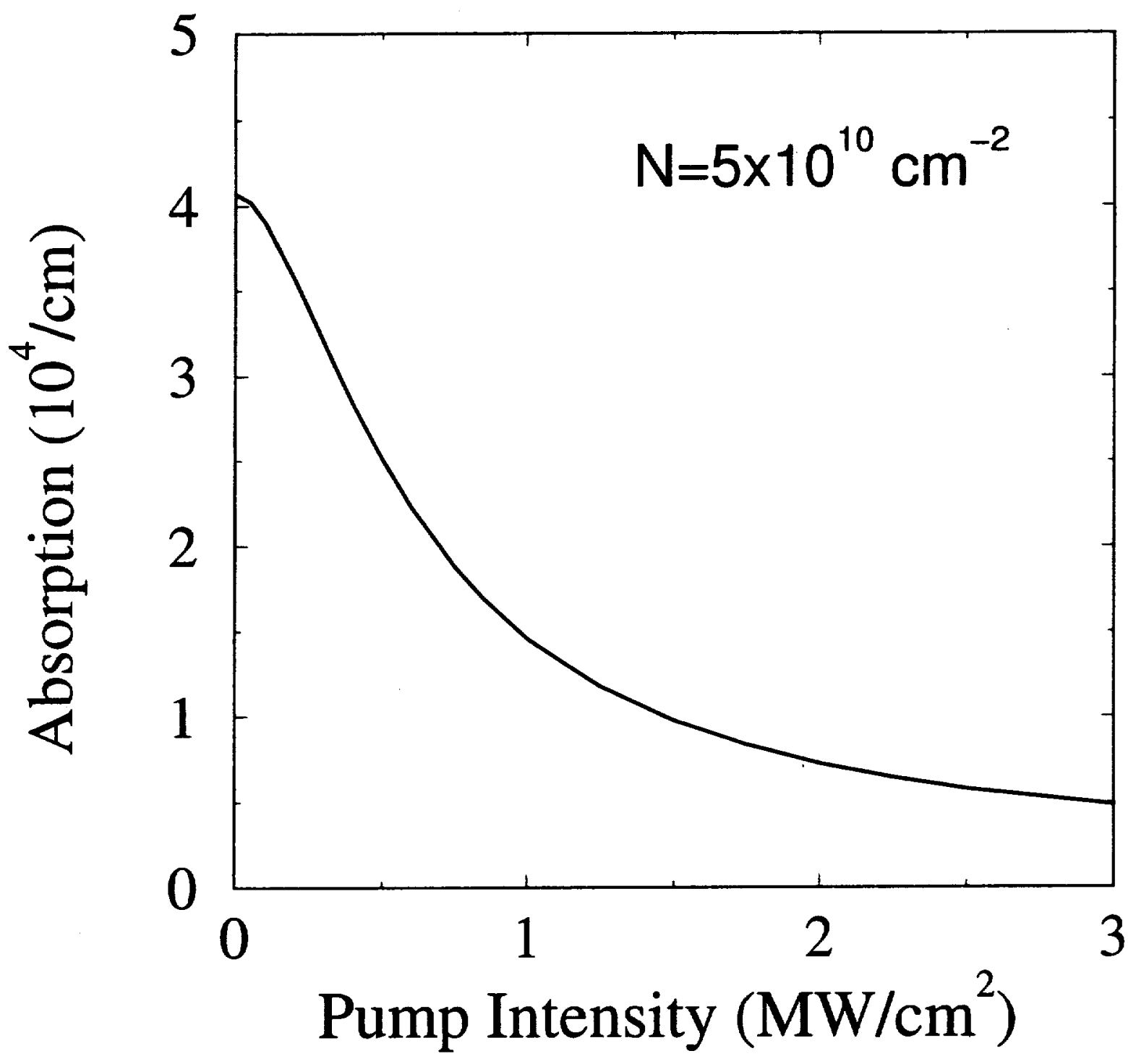

FIG. 4 


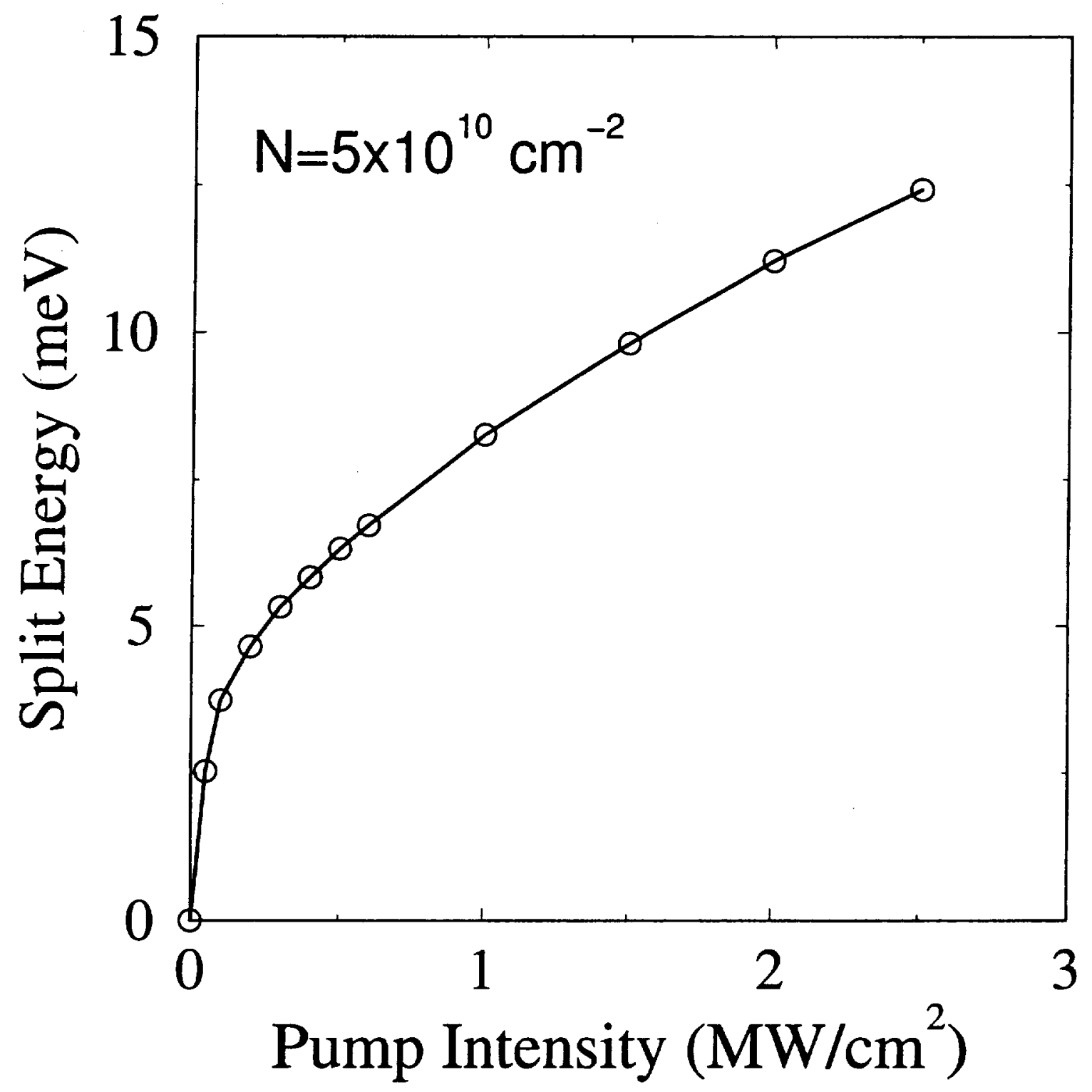

FIG. 5 


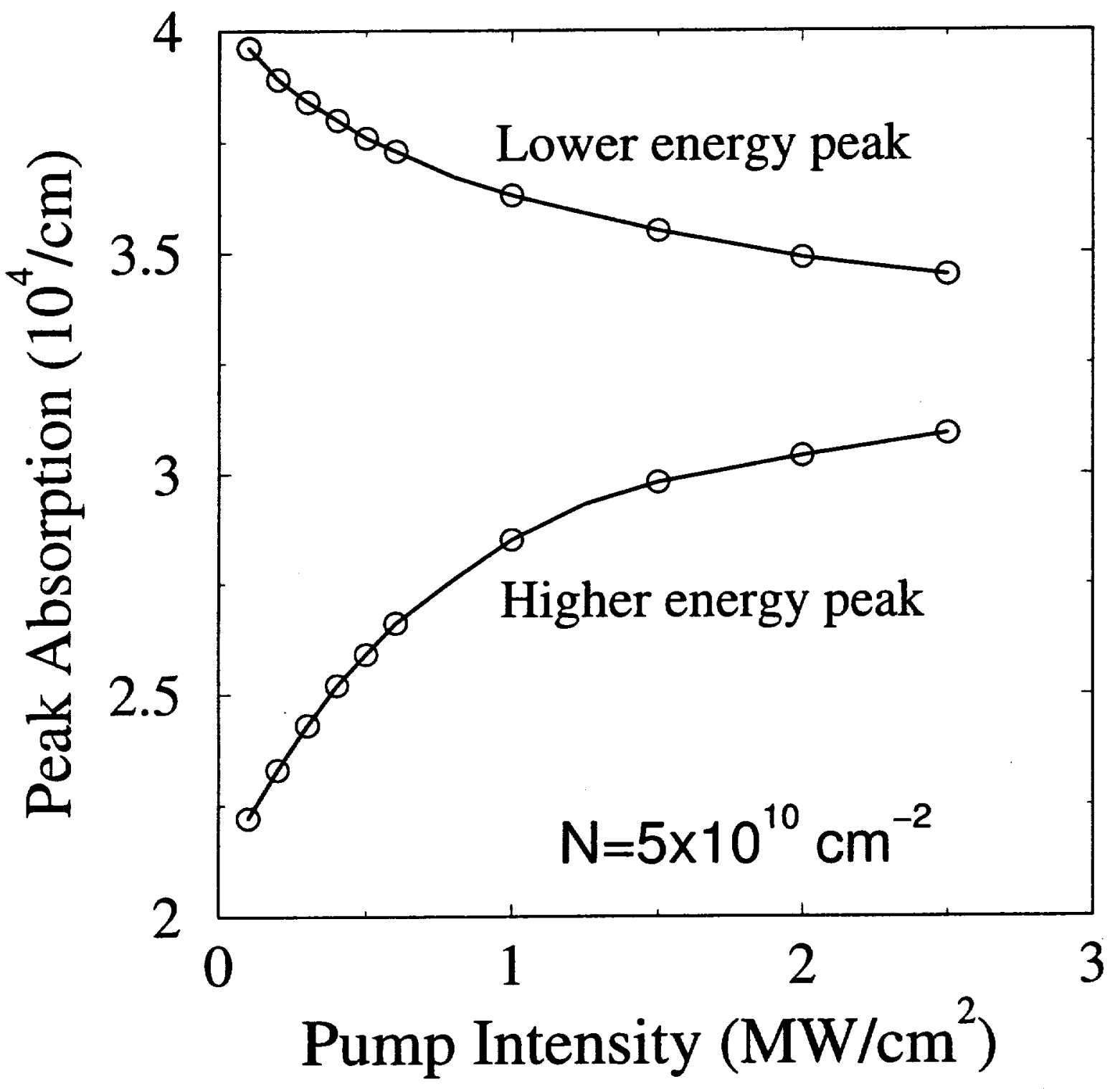

FIG. 6 


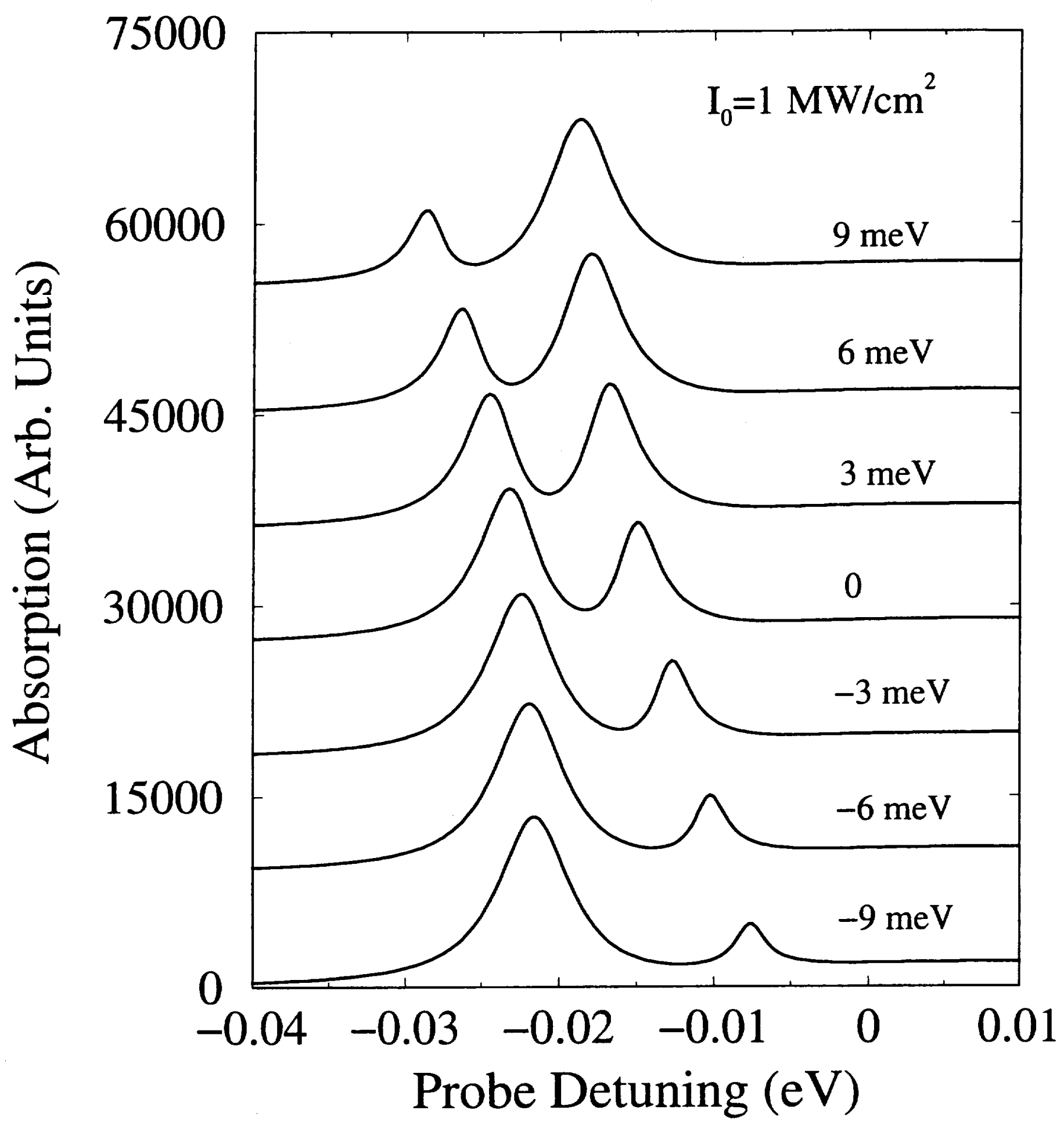

FIG. 7 


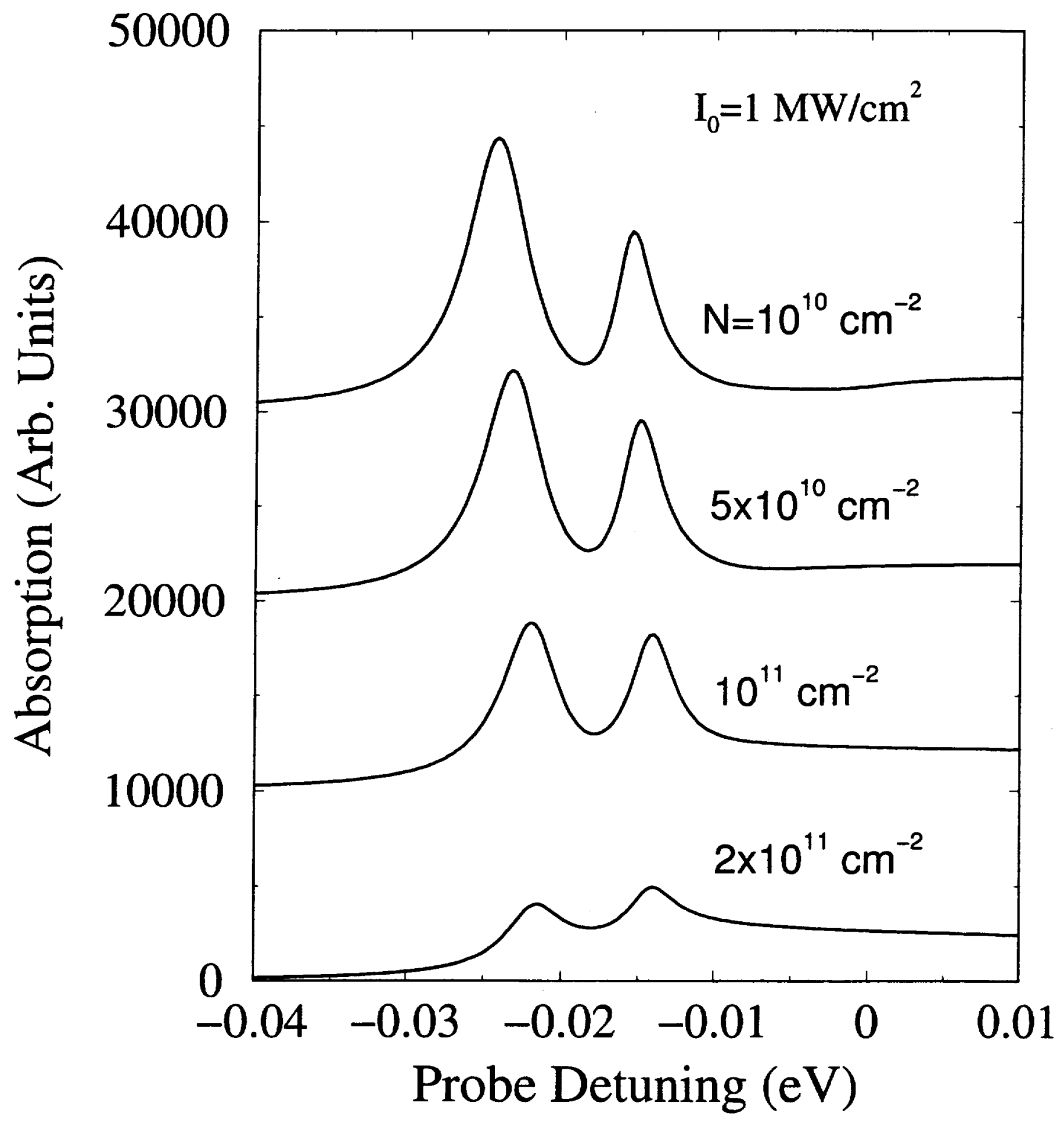

FIG. 8 
(g) The Contracting Officer may amend tasks in the same manner in which they were issued.

(h) In the event of a conflict between the requirements of the task order and the Contractor's approved task plan, the task order shall prevail.

(End of clause)

\section{H.5 EMERGENCY PREPAREDNESS AND RESPONSE (ARC52.223-90)(MAR 1999)}

In the event of an emergency that requires a Level 1,2, or 3 response, as defined in Paragraph 106, "Levels of Response," in the Ames Handbook and Emergency Preparedness Plan (AHB 1600.4), the contractor shall follow the emergency procedures (e.g., shut down equipment, conduct damage assessments, etc.) shown in Paragraph 202, "Responsibilities," of the Handbook. Responsibilities are assigned on an organizational basis; therefore, Contractors must refer to the section(s) of the Handbook that correlate with their respective COTR organization(s).

(End of Clause)

\section{H.6 DIÁSTER ASSISTANCE AND RESCUE TEAM (DART) PARTICIPATION (ARC52.223-91)(MAR 1999)}

Contractor employees are eligible to participate in the Disaster Assistance and Rescue Team (DART) if approved in writing by the Contractor and appointed by the Government. If a Contractor approves of an employee's participation, the contractor agrees to modify the employee's position description to include participation in DART, and to provide additional indemnification (e.g., worker's compensation insurance, general liability, etc.) as may be necessary to protect its employee and/or the Government while the employee is participating in the program.

\section{DART Definition}

This 90-person team is comprised of civil service, contractor, and military personnel that work at Ames Research Center and Moffett Federal Airfield. The team composition includes scientists, engineers, wind tunnel mechanics, aircraft mechanics, facility maintenance personnel, computer specialist, industrial hygienist, safety professionals, heavy equipment operators, administrative personnel, managers, procurement officials, and data specialists. DART is an umbrella organization that has six functional groups. 
